\title{
INSUFICIENCIA CARDÍACA AGUDA: FACTORES ASOCIADOS CON MORTALIDAD Hospital de San José, julio 2007 - agosto 2008 Bogotá D.C. Colombia.
}

Andrés Lancheros MD*, Yadira Valencia MD**, Walter Chaves Santiago MD***

\section{Resumen}

Introducción: la insuficiencia cardíaca (IC) es una afección que genera buen número de hospitalizaciones y cuya prevalencia se ha incrementado en las últimas décadas. Sin embargo, no se ha logrado definir un sistema de clasificación basado en los factores predictores de mal pronóstico en los pacientes que requieren hospitalización y que podrían morir durante ésta. Objetivos: describir los enfermos con falla cardíaca aguda y definir cuáles pueden ser los factores asociados con mortalidad por IC descompensada en los que ingresan a urgencias del Hospital de San José de Bogotá. Métodos: estudio descriptivo prospectivo en el período julio 2007 a agosto 2008, de los pacientes que ingresaran con IC descompensada al servicio de urgencias, evaluación de factores asociados mediante un instrumento de recolección y análisis de datos a través de medidas de asociación. Resultados: ingresaron al estudio 129 pacientes. Se encontró una mortalidad de $4,2 \%$ en el grupo con IC descompensada, valores altos de creatinina, tensión arterial sistólica elevada y sin diferencia de distribución en cuanto al género. El 50\% tenía diagnóstico de enfermedad coronaria y los hallazgos ecocardiográficos más frecuentes demostraron reducción de la fracción de eyección, disfunción diastólica e insuficiencia de la válvula mitral. Discusión: los resultados son comparables con la población mundial, pero se necesita hacer un seguimiento a mayor plazo y tener en cuenta otras variables que han demostrado estar asociadas con el desenlace fatal.

Palabras clave: insuficiencia cardíaca, mortalidad, estancia hospitalaria, factores de riesgo.

Abreviaturas: IC, insuficiencia cardíaca; EC, enfermedad coronaria; ECO, ecocardiograma(s).

\section{ACUTE HEART FAILURE: ASSOCIATED MORTALITY RISK FACTORS}

\section{San José Hospital, july 2007 - august 2008 Bogotá, Colombia.}

\section{Abstract}

Introduction: The prevalence of heart failure (HF) has increased over the last decades and causes a considerable number of hospital admissions. However, a model to identify inhospital mortality risk predictors in patients admitted with acute heart failure (AHF) is not yet available. Objectives: to describe patients with AHF and to identify which could be the

Fecha recibido: febrero 18 de 2009 - Fecha aceptado: mayo 4 de 2009

* Residente III de Medicina Interna. Fundación Universitaria de Ciencias de la Salud, Hospital de San José, Bogotá D.C. Colombia.
** Residente III de Medicina Interna. Fundación Universitaria de Ciencias de la Salud, Hospital de San José, Bogotá D.C. Colombia.

*** Instructor Asistente de Medicina Interna. Fundación Universitaria de Ciencias de la Salud, Hospital de San José, Bogotá D.C. Colombia. 
mortality risk factors on patients presenting with decompensated HF to the Emergency Room at the San José Hospital in Bogotá. Methods: this is a descriptive prospective study performed from July 2007 through August 2008, on patients presenting with decompensated HF to the ER, the assessment of associated factors was conducted through measures of association using a data collection and analysis tool. Results: 129 patients were enrolled in the study. Findings included a mortality rate of $4.2 \%$ in the decompensated HF group, increase in serum creatinine, increased systolic arterial pressure and a no gender-related difference in distribution. Fifty-percent (50\%) had a diagnosis of coronary artery disease and the most common associated echocardiographic findings revealed a decreased ejection fraction, diastolic dysfunction and mitral valve insufficiency. Discussion: these results are comparable to those of global population, but further longer follow-up is necessary considering other variables shown to be associated with a fatal outcome.

Key words: heart failure, mortality, hospital stay, risk factors

\section{Introducción}

La enfermedad cardiovascular es la principal causa de mortalidad a nivel mundial (hasta un 29\%) según los reportes de la Organización Mundial de la Salud. ${ }^{1}$ Dentro de las patologías asociadas la falla cardíaca determina un papel primordial, siendo la única de este grupo que no ha disminuido en incidencia ni mortalidad y persiste como una de las principales causas de atención en urgencias y hospitalización. ${ }^{2,3,4,5}$ La IC es un síndrome clínico en el que anomalías en la estructura o función del corazón originan incapacidad para una adecuada expulsión o llenado ventricular y así cumplir con el requerimiento metabólico del organismo. Intervienen factores neurohormonales y renales que ocasionan manifestaciones clínicas como congestión circulatoria, disnea, fatiga y debilidad. ${ }^{6}$

La IC crónica es la presentación clínica más común, de la cual se ha obtenido valiosa información para establecer la interpretación fisiopatológica de la enfermedad y la epidemiología. Por otra parte, la IC aguda (o descompensada) definida como la rápida instauración de síntomas y signos secundaria a anomalías en la función (con o sin enfermedad cardíaca previa) ${ }^{7,8}$ es frecuente, hallando en 2004 más de un millón de egresos hospitalarios en Estados Unidos y su prevalencia ha aumentado durante las últimas décadas hasta en un $175 \%$ desde 1979. La tasa de mortalidad varía de manera amplia entre 2 y $20 \%{ }^{5,8}$ A pesar de esto, la orientación investigativa ha permanecido enfocada hacia la IC crónica, de manera que pocos estudios se han realizado para analizar al paciente descompensado que requiere hospitalización. ${ }^{9}$

Evaluar al paciente con IC en el servicio de urgencias exige del médico la capacidad para detectar el amplio campo semiológico que aporta el interrogatorio y los hallazgos físicos, que se complementa con los exámenes paraclínicos y en conjunto constituyen los predictores del desenlace. La necesidad de obtener información útil para establecer factores que determinan el resultado en términos de morbimortalidad, ha generado la publicación de estudios clínicos y de bases de datos para tal fin. Empleando modelos de análisis multivariados, han mostrado mayor asociación con este desenlace: siete variables edad, sexo, frecuencia cardíaca, presión sanguínea, cifras séricas de sodio, nitrógeno ureico y creatinina, fracción de eyección del ventrículo izquierdo y clase funcional. A pesar de todo, en la literatura hasta el momento no se ha presentado un sistema de clasificación que permita establecer el riesgo de morbimortalidad y la severidad de la descompensación aguda a partir de estas variables. ${ }^{4,5,6,9}$

En la población colombiana se estima que la mortalidad por causas cardiovasculares se encuentra en 113.4 casos por 100 mil habitantes y de manera específica por falla cardíaca en 9.3 casos por 100 mil habitantes. Se han realizado pocos estudios con aceptable calidad metodológica para establecer una descripción poblacional que permita concluir asociaciones entre factores de riesgo encontrados a nivel internacional, extrapolados a nuestro país. ${ }^{10,11,12}$ A través de este estudio se describen los pacientes con falla cardíaca aguda y los posibles factores asociados con mortalidad por IC descompensada en los pacientes que ingresan al servicio de urgencias del Hospital de San José de Bogotá, en el período julio 2007 a agosto 2008 .

\section{Métodos}

El Hospital de San José es un centro de atención de IV nivel de complejidad ubicado en Bogotá, D.C, Colombia. 
Se decidió realizar en esta institución un estudio descriptivo prospectivo desde julio 2007 hasta agosto 2008, recolectando la información de los enfermos que ingresaron con criterios de falla cardíaca descompensada. Previo al inicio se estableció un protocolo que fue evaluado y aprobado por el comité de investigaciones de la Fundación Universitaria de Ciencias de la Salud.

El paciente que ingresaba a urgencias del hospital era evaluado por el grupo de turno de medicina interna que establecía el diagnóstico de IC descompensada. En pacientes mayores de quince años se definió cuando presentaban tres o más de los siguientes criterios: disnea (paroxística nocturna, ortopnea o de esfuerzo), taquicardia (frecuencia mayor de 120 por minuto) con o sin galope por tercer ruido, ingurgitación yugular, estertores a la auscultación pulmonar, edema bilateral de miembros inferiores, cardiomegalia en placas radiográficas (incremento del tamaño cardíaco en la toma de tórax), derrame pleural clínico o radiológico, edema agudo del pulmón, hepatomegalia y/o reflujo hepatoyugular.

Como criterios de exclusión se tuvieron en cuenta: aquellos remitidos de un hospital de segundo o tercer nivel sin exámenes paraclínicos al ingreso en la institución que remite (con más de 24 horas de estancia), los que solicitaran salida voluntaria en las primeras 24 horas y los enfermos que fueron remitidos a otra institución por causa médica o administrativa.

Se invitó al paciente a ingresar al estudio y se firmó el consentimiento informado previa aprobación del comité de investigación y ajustado a las normas bioéticas establecidas por la resolución 8.430 de 1993. Los datos obtenidos se registraron en un instrumento de recolección evaluado y sometido a análisis a través de una prueba piloto un mes antes del tiempo de recolección, y los mismos autores se encargaron de verificar los criterios de inclusión y exclusión. Para el estudio se consideraron las variables mortalidad, días de estancia hospitalaria, edad, sexo, así como las siguientes variables clínicas y paraclínicas documentadas al ingreso de la hospitalización: nitrógeno ureico, sodio, creatinina, presión arterial sistólica, clase funcional de base evaluada como la capacidad funcional del paciente durante el último trimestre, clase funcional al ingreso a urgencias, y frecuencias cardíaca y respiratoria. Se anotó el uso de medicamentos para control ambulatorio que consumía al menos en el último mes para manejo de IC o alguna patología cardiovascular como $\beta$-bloqueador $(\beta B)$, inhibidores de la enzima convertidora de angiotensina (IECAs), antagonistas del receptor para angiotensina (ARAs), diuréticos antagonistas de aldosterona (espironolactona) y diuréticos de asa (furosemida). Se registraron las comorbilidades, entendidas como toda enfermedad catalogada como antecedente presente o ausente al menos durante el último control ambulatorio, sin importar el tiempo del diagnóstico, tales como: diabetes mellitus 2, EC, hipertensión arterial, nefropatía diabética, insuficiencia renal crónica, enfermedad pulmonar obstructiva crónica (EPOC), hemorragia digestiva y cirrosis hepática. También se anotaron los hallazgos del ECO de quienes se les realizó el examen durante la hospitalización, independiente del momento en el cual se hizo, considerando las variables fracción de eyección (FE), hipertensión pulmonar (HTP), disfunción diastólica (DD), así como tipo, severidad y localización de cualquier valvulopatía.

El análisis estadístico de la población se realizó a través de STATA 10 ®. Las variables cuantitativas se analizaron en términos de valor máximo, mínimo, promedio y desviación estándar, y las variables cualitativas en términos de frecuencias.

\section{Resultados}

Durante trece meses de seguimiento se reunió información de 129 pacientes. Se encontró predominio de mujeres con $56 \%(n=73)$ frente a los hombres que fueron $44 \%(n=56 \%)$. El 69\% $(n=89)$ eran mayores de 65 años, con un rango de edad entre 16 y 95 años. Las características generales de la población se observan en la Tabla 1. El promedio para la creatinina al ingreso fue alto $(1.46 \mathrm{mg} / \mathrm{dl} \pm 1.36)$, al igual que el nitrógeno ureico $(30.54 \mathrm{mg} / \mathrm{dl} \pm 18.33)$. Se documentaron valores de frecuencia cardíaca y de presión arterial cercanas al límite superior de normalidad (90.51 \pm 14.8 lat/min y 135.18 $\mathrm{mm} \mathrm{Hg} \pm 28.69)$. La mayoría de los pacientes $55 \%(\mathrm{n}=71)$ tenían una clase funcional de base II que se deterioraba a III en $73 \%$. De otro lado se encontró un $34,8 \%$ de pacientes con clasificación IV al ingreso a urgencias, con una distribución similar en ambos sexos. 


\begin{tabular}{|c|c|c|c|c|c|c|c|c|}
\hline Total I 29 pacientes & \multicolumn{3}{|c|}{ Hombres } & $\%$ & \multicolumn{3}{|c|}{ Mujeres } & $\%$ \\
\hline Sexo & \multicolumn{3}{|l|}{56} & 43,41 & \multicolumn{3}{|l|}{73} & 56,59 \\
\hline Edad & \multicolumn{3}{|c|}{66,8} & $5 I, 78$ & \multicolumn{3}{|c|}{71,9} & 55,74 \\
\hline Creatinina & \multicolumn{3}{|c|}{$1,53 \pm 1,72$} & $1,40 \pm 1,0$ & & & & \\
\hline Sodio & \multicolumn{4}{|c|}{$139,2 \pm 5.2$} & \multicolumn{4}{|c|}{$138,23 \pm 6.4$} \\
\hline Nitrógeno ureico & \multicolumn{4}{|c|}{$29,09 \pm 15$} & \multicolumn{4}{|c|}{$31,94 \pm 20,57$} \\
\hline TA sistólica & \multicolumn{4}{|c|}{$|3|, \mid 4 \pm 28,99$} & \multicolumn{4}{|c|}{$138,28 \pm 28,27$} \\
\hline F. cardíaca & \multicolumn{4}{|c|}{$92,02 \pm 14,03$} & \multicolumn{4}{|c|}{$89,36 \pm 15,36$} \\
\hline F. respiratoria & \multicolumn{4}{|c|}{$21,23 \pm 3,94$} & \multicolumn{4}{|c|}{$22,79 \pm 4,29$} \\
\hline \multicolumn{9}{|l|}{ C. F NYHA Base } \\
\hline I & \multicolumn{3}{|l|}{18} & 32,14 & 11 & \multicolumn{3}{|l|}{15,07} \\
\hline ॥ & \multicolumn{3}{|l|}{23} & 41,07 & 48 & \multicolumn{3}{|l|}{65,75} \\
\hline III & \multicolumn{3}{|l|}{15} & 26,79 & 14 & \multicolumn{3}{|l|}{19,18} \\
\hline \multicolumn{9}{|l|}{ C. F NYHA Ingreso } \\
\hline ॥ & \multicolumn{3}{|l|}{11} & 19,64 & 5 & \multicolumn{3}{|l|}{6,849} \\
\hline III & 25 & & & 44,64 & 43 & 58,9 & & \\
\hline IV & 20 & & & 35,71 & 25 & 34,25 & & \\
\hline MEDICAMENTOS & Sí & $\%$ & No & $\%$ & Sí & $\%$ & No & $\%$ \\
\hline IECAs & 31 & 55,36 & 25 & 44,64 & 47 & 64,38 & 26 & 35,62 \\
\hline B. bloqueadores & 11 & 19,64 & 45 & 80,36 & 22 & 30,14 & 51 & 69,86 \\
\hline ARAs & 2 & 3,571 & 54 & 96,43 & 14 & 19,18 & 59 & 80,82 \\
\hline Espironolactona & 12 & 21,43 & 44 & 78,57 & 15 & 20,55 & 58 & 79,45 \\
\hline Furosemida & 28 & 50 & 28 & 50 & 45 & 61,64 & 28 & 38,36 \\
\hline COMORBILIDADES & Sí & $\%$ & No & $\%$ & Sí & $\%$ & No & $\%$ \\
\hline HTA & 39 & 69,64 & 17 & 30,36 & 65 & 89,04 & 8 & 10,96 \\
\hline E. coronaria & 15 & 26,79 & $4 I$ & 73,21 & 10 & 13,7 & 63 & 86,3 \\
\hline Diabetes mellitus & 13 & 23,21 & 43 & 76,79 & 28 & 38,36 & 45 & 61,64 \\
\hline Nefropatía diabética & 6 & $|0,7|$ & 50 & 89,29 & 9 & 12,33 & 64 & 87,67 \\
\hline IR crónica & 11 & 19,64 & 45 & 80,36 & 12 & 16,44 & 61 & 83,56 \\
\hline Cirrosis & I & I,786 & 55 & 98,21 & 0 & 0 & 73 & 100 \\
\hline Hemorragia digestiva alta & I & I,786 & 55 & 98,21 & 0 & 0 & 73 & 100 \\
\hline EPOC & 15 & 26,79 & $4 I$ & 73,21 & 32 & 43,84 & 41 & 56,16 \\
\hline & Nún & & & $\%$ & Nún & & & $\%$ \\
\hline MORTALIDAD & 3 & & & 50 & 3 & & & 50 \\
\hline D. DE ESTANCIA & 5.21 & & & & 6.49 & & & \\
\hline
\end{tabular}


En cuanto a la frecuencia de medicamentos utilizados durante el mes anterior y hasta el ingreso, los de mayor uso fueron los IECAs en un $60 \%(n=78)$, seguidos por furosemida en un $56.5 \%(n=73)$ y solo un $12,4 \%(n=16)$ recibían ARAs.

Las comorbilidades de mayor prevalencia al ingreso de la hospitalización fueron la hipertensión arterial, seguida del EPOC y en tercer lugar EC. Dos episodios aislados de cirrosis y hemorragia digestiva alta se encontraron en los hombres. La Tabla 2 describe la frecuencia de comorbilidades en la población general y fallecida.

Se realizaron 64 ecocardiogramas y sus hallazgos más relevantes se informan en la Tabla 3. En el 82\% se documentó una fracción de eyección $=50 \%$ y la gran mayoría de los reportes informó disfunción diastólica (94\%). La hipertensión arterial estuvo presente en un 53\% y se evidenció una mayor frecuencia de compromiso valvular mitral por insuficiencia.

Hubo una mortalidad del 4,6\% $(n=6)$ con igual distribución por género (tres mujeres y tres hombres). $67 \%$ $(n=4)$ eran mayores de 65 años, $67 \%(n=4)$ tenían creatinina elevada al ingreso (mayor $1.3 \mathrm{mg} / \mathrm{dl}$ ) y $83 \%(n=5)$ con cifras de presión arterial sistólica mayores de $140 \mathrm{~mm} \mathrm{Hg}$. Todos presentaron frecuencia cardíaca mayor a 90 lat $/ \mathrm{min}$, el 33\% $(\mathrm{n}=2)$ ingresó con clase funcional IV y el resto presentó deterioro a clase funcional III. Respecto al tratamiento, el $67 \%$ se encontraba en manejo, al menos durante el último mes, con IECAs, $50 \%$ con furosemida, $33 \%$ con âB, $17 \%$ con espironolactona y ninguno de ellos recibía ARAs. Las comorbilidades más frecuentes fueron hipertensión arterial (83\%) y EC (50\%). Se realizó ECO en dos pacientes encontrando en ambos: disfunción diastólica, y sistólica con fracción de eyección menor de 55\% (10 y 25\% respectivamente), hipertensión pulmonar severa e insuficiencia mitral moderada a severa. Dos pacientes presentaron estancia hospitalaria de diez días.

La Figura 1 describe la distribución de la estancia hospitalaria para la población general, resaltando que la mayoría (58\%) tenía duración menor de cinco días.

\section{Discusión}

En el último decenio la IC ha jugado un papel importante en el aumento de la morbimortalidad de la enfermedad cardiovascular, debido al incremento de la expectativa de vida y la frecuencia de comorbilidades en estos pacientes, lo que implica un mayor riesgo de requerir hospitalización por descompensación. Al igual que para el estudio y manejo de otras afecciones, la IC descompensada debería ser evaluada en términos de estratificación de acuerdo con el establecimiento de factores predictores de morbimortalidad que puedan servir al clínico para definir una clara opción terapéutica.

\begin{tabular}{|l|c|c|c|}
\hline \multicolumn{2}{|c|}{ Tabla 2. Comorbilidades y mortalidad en IC descompensada } \\
\hline Comorbilidades & Frecuencia & $\%$ & Muerte \\
\hline Hipertensión arterial & 104 & 80,62 & 5 \\
E. coronaria & 25 & 19,38 & 3 \\
D. mellitus & 41 & 31,78 & 2 \\
Nefropatía diabética & 15 & 11,63 & 1 \\
I. renal crónica & 23 & 17,83 & 2 \\
Cirrosis & 1 & 0,78 & 0 \\
H.V. digestivas & 2 & 1,55 & 1 \\
EPOC & 47 & 36,43 & 2 \\
\hline
\end{tabular}



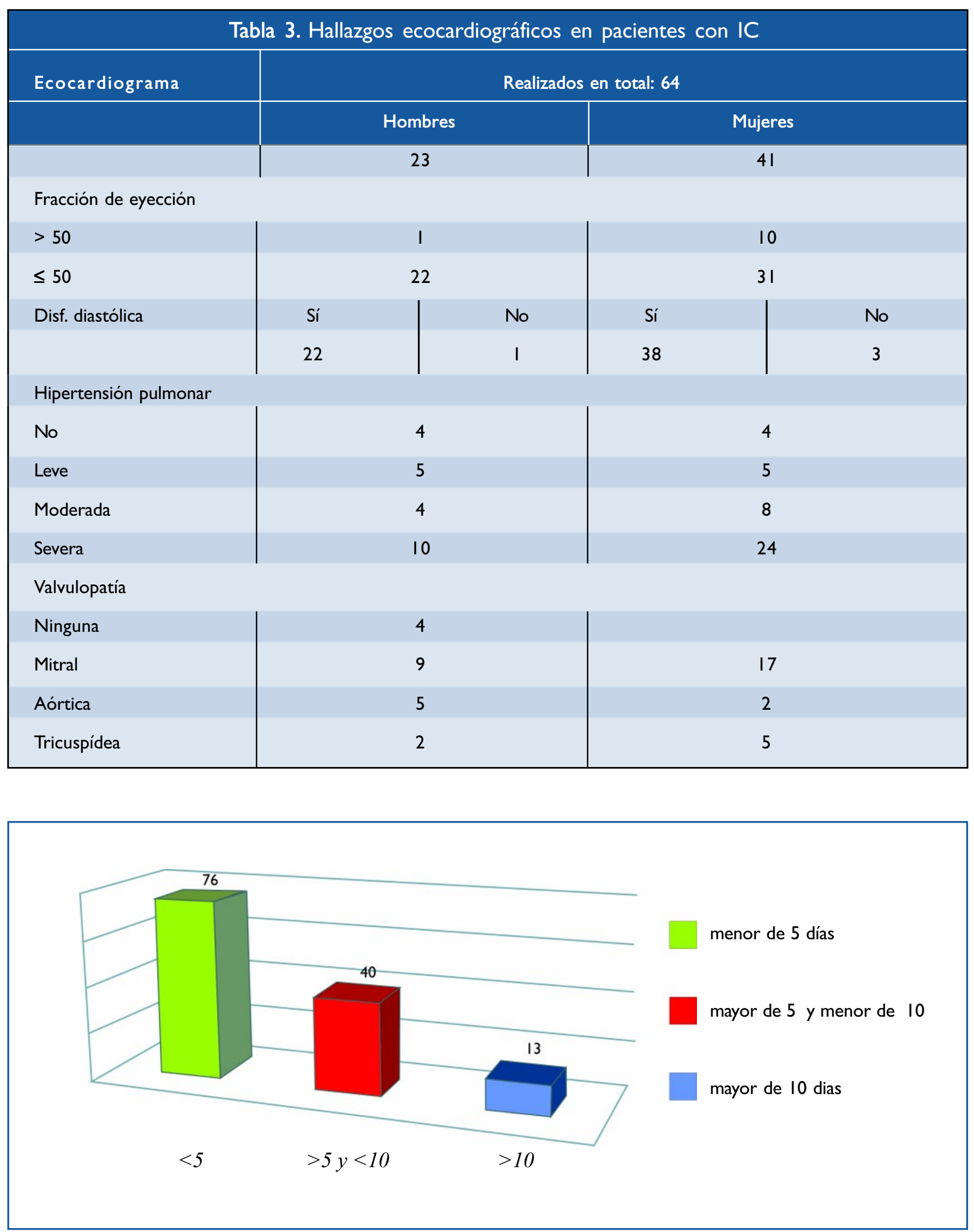

Figura I. Estancia hospitalaria de los pacientes con IC. 
Los estudios internacionales de mayor impacto demográfico y epidemiológico se apoyan en análisis de bases de datos. ${ }^{5,8,9,13,14}$ El registro IMPACT-HF, fue un estudio prospectivo multicéntrico realizado en Estados Unidos cuyo reporte incluyó la descripción de las características clínicas de pacientes hospitalizados con IC y el seguimiento a 60 días valorando las frecuencias de mortalidad y de reingreso. ${ }^{15} \mathrm{La}$ base de datos ADHERE con más de 100.000 registros, posee alrededor de 80 variables de recolección, con importantes resultados obtenidos a partir de subestudios como la descripción poblacional, el manejo terapéutico endovenoso temprano y los datos paraclínicos como valores predictores. ${ }^{13}$ El estudio EURO HEART es una base de datos con alrededor de 46 mil fallecimientos de los cuales 11.327 fueron por IC y describe las variables en forma retrospectiva. ${ }^{14} \mathrm{El}$ estudio OPTIMIZE presenta un análisis de variables de pacientes hospitalizados con IC y su seguimiento a siete meses, valorando mortalidad y reingreso ${ }^{16}$ (Tabla 4).

Los análisis de estos registros informan que entre 65 y $87 \%$ de los que ingresan a urgencias con IC tienen dentro de sus características principales el deterioro en su clase funcional, que concuerda con el presente estudio (73\% de los pacientes con CFb II pasaban a CFi III). Las características demográficas sexo y edad fueron si- milares a los estudios mencionados ${ }^{5,8,9,13,14,16}$ de tal forma que se encontró un predominio femenino en la población, pero la distribución de los fallecidos no mostró diferencias en cuanto al género. La edad promedio fue de 70 años, que se correlaciona con el promedio reportado a nivel internacional, valor esperado por el proceso evolutivo de la enfermedad.

Las comorbilidades de mayor prevalencia en el estudio para ambos sexos fueron la hipertensión arterial, seguida de EPOC y en tercer lugar EC, presentándose esta última con mayor frecuencia en hombres que en mujeres. Estos hallazgos difieren de los reportes, pues la EC ocupa el segundo lugar dentro de las comorbilidades asociadas con IC. Estos resultados pueden estar relacionados con un subregistro de la EC pues al ser tomada como un antecedente al ingreso, probablemente no se realizó una búsqueda exhaustiva durante la hospitalización. Además, cierto número de pacientes con infarto agudo de miocardio fue excluido por remisión temprana y no fueron analizados.

Llama la atención que más del $70 \%$ de los ingresados no recibían un tratamiento ambulatorio óptimo como recomiendan las guías de manejo internacionales. ${ }^{7}$ Este hallazgo puede estar relacionado con una inadecuada adherencia de los pacientes a sus medicamentos y/o

Tabla 4. Relación entre los estudios internacionales de pacientes con IC

\begin{tabular}{|l|c|c|c|c|}
\hline & ADHERE & EURO HF & OPTIMIZE-HF & H. de San José \\
\hline & $(\mathrm{N}=107920)$ & $(\mathrm{N}=1$ I 327) & $(\mathrm{N}=34059)$ & $(\mathrm{N}=129)$ \\
\hline Promedio edad (años) & 75 & 71 & 73 & 70 \\
Mujeres (\%) & 52 & 47 & 52 & 56 \\
IC previa (\%) & 75 & 65 & 87 & $\mathrm{NR}$ \\
Fracción de eyección (\%) & $5 \mathrm{I}$ & 46 & 52 & 37 \\
EC (\%) & 57 & 68 & 50 & 85 \\
Hipertensión arterial (\%) & 72 & 53 & 71 & 31 \\
Diabetes (\%) & 44 & 27 & 42 & $\mathrm{NR}$ \\
Fibrilación atrial & 31 & 43 & 31 & 18 \\
Insuf. renal crónica & 30 & 18 & $\mathrm{NR}$ & \\
\hline
\end{tabular}


con la no aplicación de las recomendaciones dadas por las guías.

Dentro de los exámenes descritos es importante recalcar que el uso del ECO apenas sobrepasa el 50\% de los datos analizados, ya sea porque algunos tenían ECO previo por lo cual el clínico prescindía de éste durante la evaluación en urgencias y en la hospitalización. Sin embargo, el ECO ha demostrado ser una herramienta útil y objetiva en la valoración del paciente con IC, que está disponible a nivel local y se recomienda su utilización frecuente.

El objetivo primordial del estudio fue valorar los factores asociados con mortalidad intrahospitalaria, encontrando una frecuencia de 4,6\% (seis pacientes). Estos resultados son comparables con la tasa descrita en reportes internacionales. ${ }^{5,8}$ Sin embargo, este porcentaje representa una baja frecuencia para el número de pacientes incluidos en el estudio, lo que no permitió realizar análisis de asociación entre las variables y el desenlace. A pesar de esta consideración, los pacientes que murieron tenían características comunes a los informes mundiales: edad promedio similar, frecuencia de comorbilidades (hipertensión arterial seguida de EC, EPOC e insuficiencia renal crónica), deterioro en la clase funcional y los hallazgos del ECO.

Este estudio proporciona información acerca de la epidemiología local de la IC en un hospital de IV nivel de complejidad de la ciudad de Bogotá. Considerando la publicación de otros trabajos a nivel nacional, ${ }^{10}$ se cuenta con los recursos necesarios en la institución para una adecuada evaluación clínica y paraclínica de los pacientes. La utilización de estas herramientas podría reflejarse en mejores medidas terapéuticas, optimizando así la evolución clínica y reduciendo el número de complicaciones y costos. No fue posible demostrar una asociación entre los factores de riesgo y la mortalidad por falla cardíaca por el bajo tamaño de la muestra. Sin embargo, se genera el camino para iniciar una línea de investigación en IC en el servicio de medicina interna. Como medidas para tener en cuenta en la continuidad de esta investigación se sugiere ampliar la base de datos, adicionar el seguimiento de la población luego de su egreso, considerar la mortalidad intrahospitalaria y ambulatoria, enfatizar en las características de las comorbilidades y del manejo terapéutico ambulatorio, evaluar otras variables paraclínicas descritas en la literatura y que han demostrado ser factores predictores, dentro de los cuales se podrían incluir: hallazgos electrocardiográficos (arritmias), valores de hemoglobina, proteína $\mathrm{C}$ reactiva y péptidos natriuréticos.

\section{Referencias}

1. Organización Mundial de la Salud. Enfermedades cardiovasculares [monografía en internet]. Nueva York: OMS; 2007 [citado 30 Jun. 2009]. Disponible en: http:/ /www.who.int/mediacentre/factsheets/fs317/es/ index.html

2. Luthi JC, McClellan WM, Fitzgerald D, Krumholz HM, Delaney RJ. Multi-State Collaborative Congestive Heart Failure Study Group. Mortality associated with the quality of care of patients hospitalized with congestive heart failure. Int J Qual Health Care. 2002; 14(1):15-24

3. McKee PA, Castelli WP, McNamara PM. The natural history of congestive heart failure: the Framingham study; N Engl J Med. 1971; 285(6):1441-46.

4. Cortina A, Cortina R. Insuficiencia cardíaca: marcadores pronósticos de mortalidad. Cardiovasc Risk Factors. 2001; 10(1): 15-20

5. Fonarow GC, Adams KF Jr, Abraham WT, Yancy CW, Boscardin WJ. ADHERE Scientific Advisory Committee. Study Group, and Investigators. Risk stratification for in-hospital mortality in acutely decompensated heart failure: classification and regression tree analysis. JAMA 2005; 293(5):572-80.

6. Teo K, Montague T, Ackman M. Clinical quality improvement network investigators: mortality risk and patterns of practice in 4606 acute care patients with congestive heart failure: the relative importance of age, sex, and medical therapy. Arch Intern Med. 1996; 156(15): 1669-73.

7. Nieminen MS, Bohm M, Cowie MR. Executive summary of the guidelines on the diagnosis and treatment of acute heart failure: the task force on acute heart failure of the European Society of Cardiology. Eur Heart J. 2005; 26(3):384-416.

8. Fonarow GC. Epidemiology and risk stratification in acute heart failure. Am Heart J. 2008; 155(4):200-7. 
9. Abraham WT, Fonarow GC, Albert NM. Predictors of in-hospital mortality in patients hospitalized for heart fFailure. J Am Coll Cardiol. 2008; 52(4): 347-56.

10. Ospina AV, Gamarra HG. Características clínicas y epidemiológicas de la insuficiencia cardiaca en el Hospital Universitario Ramón González Valencia de Bucaramanga, Colombia. Salud UIS. 2004; 36(2):125-31.

11. García R, Casas JP, Silva F. Factores predictores de mortalidad en pacientes con insuficiencia cardiaca. Acta Med Colomb. 2002; 27(4):322-31.

12. Colombia. Ministerio de Protección Social. Situación de salud en Colombia: indicadores básicos [monografía en Internet]. Bogotá : El Ministerio; 2007 [citado 30 Jun. de 2009]. Disponible en: http:// www.minproteccionsocial.gov.co/vBecontent/ NewsDetail.asp?ID=15895\&IDCompany $=3$

13. Yancy CW, Lopatin M. Clinical presentation, management and in-hospital outcomes of patients admitted with acute decompensated heart failure with preserved systolic function a report from the Acute Decompensated Heart Failure National Registry (ADHERE) Database. J Am Coll Cardiol. 2006; 47(2): 76-84.

14. Cleland JGF, Swedberg K, Follath F, Komajda M, Cohen-Solal A, Aguilar A, Dietz R, Gavazzi A. The EuroHeart Failure Survey programme - a survey on the quality of care among patients with heart failure in Europe. Part 2: treatment. Eur. Heart J. 2003; 24(5):442-63.

15. O'Connor CM, Stough WG. Demographics, clinical characteristics, and outcomes of patients hospitalized for decompensated heart failure: observations from the IMPACT-HF registry. J Card Fail. 2005 Apr; 11(3):200-5

16. Fonarow GC, Abraham WT, Albert NM, et al. Organized Program to Initiate Lifesaving Treatment in Hospitalized Patients with Heart Failure (OPTIMIZE-HF): rationale and design. Am Heart J 2004; 148(1): 43-51. 